\title{
Política de antibióticos en pacientes críticos
}

\author{
F. Álvarez Lerma ${ }^{a, *}$, R. Sierra Camerino ${ }^{b}$, L. Álvarez Rocha $^{c}$ y Ó. Rodríguez Colomo ${ }^{d}$
}

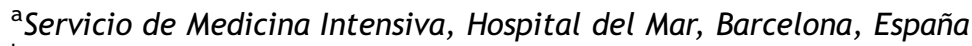

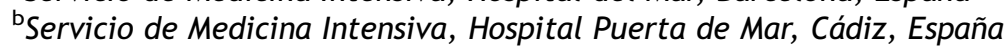

'Servicio de Medicina Intensiva, Hospital Juan Canalejo, A Coruña, España

${ }^{\mathrm{d} S e r v i c i o ~ d e ~ M e d i c i n a ~ I n t e n s i v a, ~ H o s p i t a l ~ C l i ́ n i c o ~ U n i v e r s i t a r i o ~ d e ~ V a l e n c i a, ~ V a l e n c i a, ~ E s p a n ̃ a ~}$

Recibido el 17 de diciembre de 2009; aceptado el 5 de marzo de 2010

Disponible en Internet el 13 de mayo de 2010

\section{PALABRAS CLAVE \\ Política de \\ antibióticos; \\ Desescalada \\ teraupética; \\ Ciclado de \\ antibióticos; \\ Tratamiento \\ anticipado}

\section{KEYWORDS}

Antibiotic policy;

Therapeutic

de-escalation;

Cycling of antibiotics;

Preemptive treatment

\begin{abstract}
Resumen
El conjunto de normas y estrategias desarrolladas para mejorar y optimizar el empleo de los antimicrobianos recibe el nombre de política de antibióticos. Los pacientes críticos ingresados en servicios de medicina intensiva presentan unas características especiales (gravedad, agentes patógenos, alteración de órganos o sistemas) que justifican el empleo de los antibióticos de forma diferencial al de otros pacientes hospitalizados. La influencia y el impacto de los antibióticos se observa en los pacientes que los reciben (respuesta clínica, evolución) y en el ecosistema que rodea al paciente (flora hospitalaria). Este impacto es especialmente visible en los pacientes críticos y en la flora endémica de las unidades de cuidados intensivos.

En este artículo se describe un conjunto de normas (decálogo de normas) y estrategias (desescalada terapéutica, ciclado de antibióticos, tratamiento anticipado y parámetros farmacocinéticos/farmacodinámicos) que se han aplicado y desarrollado en los pacientes críticos para optimizar el empleo de los antimicrobianos con el objetivo de conseguir la máxima efectividad y la mínima morbilidad.
\end{abstract}

(c) 2009 Elsevier España, S.L. y SEMICYUC. Todos los derechos reservados.

Antibiotic policy in critical patients

\begin{abstract}
The combination of guidelines and strategies developed to improve and optimize the use of antimicrobials receives the name of antibiotic policy. Critical patients admitted to the Intensive Medicine Services have special characteristics (severity, pathogen agents, organ and/or system disorders) that justify the use of antibiotics differentially than for other hospitalized patients. The influence and impact of the antibiotics are observed in the patients who receive them (clinical response, course) and in the ecosystem surrounding
\end{abstract}

\footnotetext{
*Autor para correspondencia.

Correo electrónico: Falvarez@imas.imim.es (F. Álvarez Lerma).
} 
the patient (hospital flora). This impact is especially visible in the critical patients in the endemic flora of the ICU.

This article describes a combination of guidelines (guideline decalogue) and strategies (therapeutic de-escalation, cycling of antibiotics, preemptive treatment and pharmacokinetic/pharmacodynamic parameters) that have been applied and developed in the critical patients to optimize the use of the antimicrobials in order to achieve the maximum effectivity and minimum morbidity.

(c) 2009 Elsevier España, S.L. and SEMICYUC. All rights reserved.

«La prescripción de antibióticos no debe ser nunca un acto rutinario sino que debe estar precedida, en todos los casos, de actos de reflexión, antes, durante y después de su administración».

\section{Introducción}

Los antimicrobianos son fármacos utilizados con gran frecuencia en los servicios o unidades de cuidados intensivos (UCI). En la última década se ha demostrado que la administración precoz de antimicrobianos con espectro adecuado influye a corto plazo en una evolución favorable de los pacientes críticos $^{1-4}$, mientras que a largo plazo, los antimicrobianos favorecen la aparición de flora emergente y condicionan cambios en las resistencias en aquellos patógenos que forman parte del ecosistema de los hospitales ${ }^{5,6}$. A lo largo de los años, se ha propuesto un conjunto de normas y estrategias para mejorar y optimizar su empleo ${ }^{7-9}$, lo que en conjunto recibe el nombre de política de antibióticos.

Para desarrollar en un hospital un programa de política de antibióticos es necesario la participación activa de todo el personal sanitario, tanto la de aquellos dedicados al control y la vigilancia de las infecciones relacionadas con la asistencia sanitaria (antes llamadas infecciones nosocomiales) como la de los dedicados a su prevención o tratamiento.

Tabla 1 Decálogo de normas de política de antibióticos en pacientes críticos

1 Utilizar antibióticos solo cuando existe la sospecha clínica o microbiológica de una infección

2 Obtener muestras de los tejidos infectados antes de iniciar un tratamiento con antibióticos

3 Elegir los antibióticos empíricos utilizando protocolos terapéuticos consensuados

4 Obtener una respuesta rápida del laboratorio de microbiología

5 Seleccionar un tratamiento dirigido cuando se conozca la etiología de la infección

6 Monitorizar la eficacia del tratamiento

7 Vigilar la aparición de efectos secundarios o flora emergente multirresistente

8 Limitar la duración del tratamiento en función de la respuesta clínica o microbiológica

9 Responsabilizar a un médico intensivista del control, la vigilancia y el tratamiento de las infecciones

10 Corresponsabilizar a todo el equipo médico de la necesidad del cumplimiento de las normas de política de antibióticos
En algunas $\mathrm{UCl}$ existen médicos que emplean parte de su tiempo en funciones de control, vigilancia, tratamiento y prevención de las infecciones relacionadas con la asistencia sanitaria y controlan la utilización de los antibióticos, lo que ha supuesto un impulso a la consolidación de la política de antibióticos en estas áreas de alto riesgo. En colaboración con otras especialidades básicas (microbiología, farmacia, farmacología, medicina preventiva) son los responsables de diseñar las estrategias terapéuticas más adecuada a la situación de cada UCI.

En este artículo se incluye un conjunto de normas que, a juicio de los autores de este documento, son básicas para la utilización de antibióticos en pacientes críticos (tabla 1) así como aquellas estrategias que se han propuesto con la finalidad de optimizar su empleo y disminuir la morbilidad relacionada con su uso. Todo esto constituye la base de la política de antibióticos y del uso racional de los antimicrobianos en cualquier UCl. Para su elaboración no se ha seguido ninguna metodología específica, por lo que no se incluye ningún tipo de graduación de evidencia ni de recomendación.

\section{Normas básicas del uso de antimicrobianos en pacientes críticos. Decálogo de normas}

\section{Primera norma. «Utilizar antibióticos solo cuando existe la sospecha clínica o microbiológica de una infección»}

Los antibióticos solo deben utilizarse, con finalidad terapéutica, cuando existe la sospecha clínica o microbiológica de infección, aunque en los pacientes críticos puede ser difícil diferenciar entre sepsis (respuesta inflamatoria sistémica frente a la infección) y síndrome de respuesta inflamatoria sistémica frente a otros estímulos inflamatorios de naturaleza no infecciosa (traumatismo, poliartritis, pancreatitis, hemorragia, entre otras) y que, inicialmente, cursan con la misma expresividad clínica ${ }^{10,11}$. Asimismo, no debe ser motivo de inicio de tratamiento antibiótico el aislamiento de microorganismos en algunas muestras (esputo, aspirado traqueal, heces, piel) en las que existe de forma habitual una flora endógena o el aislamiento en sangre o en muestras pulmonares, incluso en las obtenidas con métodos invasivos (catéter telescopado protegido, lavado broncoalveolar, etc.) de patógenos escasamente virulentos (Staphylococcus coagulasa negativos, Corynebacterium sp.). En todos los casos, es preciso razonar y relacionar la situación clínica del paciente con los hallazgos microbiológicos. 
En los casos de sospecha clínica de infección respiratoria de vías bajas (esputo purulento, leucocitosis, fiebre), sin coexistencia de infiltrados radiológicos, en pacientes con ventilación mecánica es conveniente la realización de exploraciones complementarias para confirmar el diagnóstico de infección. En los pacientes con fiebre de foco desconocido, siempre que no haya una respuesta sistémica grave, el recambio o la retirada de catéteres puede ser suficiente para solucionar el proceso infeccioso. La administración de antibióticos, sin esperar la respuesta al cambio de los catéteres, es uno de los motivos por los que ha aumentado el consumo de glucopéptidos en las áreas de cuidados intensivos.

Una parte importante de los antimicrobianos utilizados en las $\mathrm{UCl}$ se prescriben como profilaxis. En estos casos se recomienda su uso de acuerdo con protocolos consensuados en el hospital y por cortos períodos de tiempo. Existen estudios que demuestran la eficacia de la administración de antibióticos locales no absorbibles en el tubo digestivo y en la orofaringe para prevenir la aparición de infecciones endógenas tardías en pacientes con ventilación mecánica ${ }^{12-16}$ o la administración de antibióticos sistémicos para prevenir la aparición de infecciones respiratorias precoces en pacientes en coma que precisan intubación de la vía aérea ${ }^{17}$. A pesar de las numerosas evidencias acumuladas, la utilización de antibióticos locales no se ha generalizado en las $\mathrm{UCl}$ y se ha reservado su empleo en pacientes de riesgo (trasplante hepático o pulmonar, traumáticos graves o quirúrgicos complejos ${ }^{18-20}$. En pacientes con pancreatitis aguda grave se había recomendado hasta hace poco el empleo de antibióticos para prevenir la aparición de complicaciones infecciosas ${ }^{21}$; sin embargo, estudios recientes, mejor diseñados, han demostrado su falta de efectividad ${ }^{22}$.

\section{Segunda norma. «Obtener muestras de los tejidos infectados antes de iniciar un tratamiento con antibióticos»}

En los pacientes críticos, la prescripción de antibióticos sin obtener muestras de los tejidos infectados y de sangre no está justificada en ninguna ocasión. Antes de administrar la primera dosis hay que hacer todo lo posible para obtener muestras para cultivos (incluidas al menos 2 muestras de sangre), siempre que no se retrase la administración del antibiótico. El aislamiento de agentes patógenos permite confirmar la infección en determinadas situaciones clínicas en las que pueden existir dudas diagnósticas (bacteriemia, neumonía, infección del tracto urinario). Por eso, el inicio de un tratamiento con antibióticos debe ir precedido, en todos los casos, de la obtención de muestras adecuadas de cada foco y se deben utilizar, en los casos necesarios, técnicas invasivas e incluso quirúrgicas. Cuando no sea posible la utilización de procedimientos seguros para la obtención de muestras en los se requiere la colaboración de otros especialistas, se deben obtener muestras consideradas menos eficaces o con menos seguridad diagnóstica, como las secreciones traqueales (aspiración traqueal simple), el exudado abdominal (drenajes, fístulas o heridas externas) o el exudado orofaríngeo, nasal o rectal. La utilización de técnicas invasivas «ciegas» (catéter telescopado, lavado broncoalveolar), sobre todo las protegidas frente a la contaminación, ha demostrado ser de utilidad para el diagnóstico de infecciones respiratorias ${ }^{23,24}$.

Si el paciente está utilizando antibióticos, en el momento de detectarse una nueva infección deben tomarse las muestras con la máxima rapidez, sin esperar a que disminuya la acción de los antibióticos circulantes, ya que es muy posible que los patógenos causantes de la infección sean resistentes a los antibióticos que recibe. En estos casos se aconseja la obtención de las muestras en el momento previo a la administración de la siguiente dosis del antibiótico, que se corresponde con su mínima concentración plasmática.

\section{Tercera norma. «Elegir los antibióticos empíricos utilizando protocolos terapéuticos consensuados»}

Los antibióticos empíricos que se utilizan en la mayoría de los procesos infecciosos diagnosticados en pacientes críticos deben estar incluidos en los protocolos de actuación, previamente elaborados en cada $\mathrm{UCl}$. En la tabla 2 se incluyen aquellos procesos infecciosos en los que es aconsejable disponer de protocolos terapéuticos empíricos en las $\mathrm{UCl}$.

Los protocolos contemplan diferentes situaciones clínicas (tratamiento de primera y segunda elección, tratamiento de rescate), incluyen algunas situaciones especiales de los pacientes (insuficiencia renal, alergia a betalactámicos, embarazo)

y recomiendan los antibióticos, dosis y vías de administración más adecuados para el tratamiento de los patógenos esperados en cada zona geográfica, hospital o UCI. En los pacientes críticos, en todos los casos se empleará la vía intravenosa para asegurar, lo antes posible, una elevada concentración plasmática y tisular; se respetará la dosificación máxima recomendada, que se asocia con concentraciones plasmáticas óptimas, y se realizarán los ajustes necesarios según la función renal de los pacientes.

En la elaboración de los protocolos es aconsejable que intervengan todos los especialistas comprometidos en la prevención y el tratamiento de las infecciones (microbiólogos, farmacéuticos, farmacólogos, infectológos, preventivistas e intensivistas), aunque la responsabilidad de su aplicación, así como la realización de auditorías sobre su

Tabla 2 Procesos infecciosos frente a los que es aconsejable disponer de protocolos terapéuticos específicos

1. Neumonía comunitaria grave

2. Meningitis aguda

3. Encefalitis

4. Sepsis de origen urinario

5. Peritonitis secundaria

6. Neumonía nosocomial

7. Infección urinaria relacionada con sonda uretral

8. Meningitis posquirúrgica

9. Peritonitis posquirúrgica

10. Infección relacionada con catéter

11. Sepsis grave sin focalidad 
cumplimiento, corresponde al médico de medicina intensiva experto en enfermedades infecciosas.

La información periódica de la frecuencia de los agentes patógenos que predominan en las muestras orgánicas más significativas, así como conocer su sensibilidad a los antimicrobianos, permite modificar los protocolos terapéuticos empíricos y ajustarlos a la realidad epidemiológica de cada área. La colaboración y la comunicación con el servicio de microbiología son fundamentales para readaptar los protocolos periódicamente.

\section{Cuarta norma. «Lograr una respuesta rápida del laboratorio de microbiología»}

El conocimiento de los patógenos causantes de una determinada infección o de su sensibilidad facilita el empleo de antibióticos de manera dirigida, y evita que los tratamientos empíricos de amplio espectro se mantengan muchos días o incluso hasta el final del tratamiento. Para esto, es necesario optimizar el traslado de muestras al laboratorio, la organización de su procesamiento y la difusión de sus resultados.

El manejo de las muestras debe seguir unas normas previamente establecidas, que incluyen la técnica de extracción u obtención de cada muestra, los medios de transporte y los tiempos mínimos en que deben llegar a los laboratorios para su procesamiento ${ }^{25}$. El incumplimiento de alguna de ellas puede influir en la calidad de los resultados, ya que se aumenta el riesgo de contaminación y se facilita, en algunos casos, la multiplicación in situ, lo que distorsiona el resultado de estudios cuantitativos.

En los últimos años se han incorporado nuevas técnicas de diagnóstico rápido, basadas en el diagnóstico molecular (PCR en tiempo real) $^{26-28}$. Es aconsejable la incorporación progresiva de estos métodos de trabajo en los laboratorios de microbiología, ya que un diagnóstico etiológico precoz favorece la utilización de los antibióticos más adecuados y específicos.

La información obtenida en los laboratorios de microbiología, incluso los resultados de la técnica más sencilla (tinción de Gram), debe llegar con rapidez a los clínicos responsables del paciente. La comunicación mediante correo electrónico ha disminuido los tiempos de respuesta, pero este sistema no está disponible en todos los hospitales. El contacto telefónico para informar de aquellos resultados de mayor relevancia así como del aislamiento de patógenos multirresistentes (identificados como marcadores de multirresistencia) es la alternativa más sencilla y eficaz.

En algunos hospitales, los médicos intensivistas responsables de la vigilancia-control de infecciones relacionadas con la asistencia sanitaria participan diariamente en reuniones con el servicio de microbiología, lo que facilita el intercambio de información y el conocimiento de los cambios en los patrones de resistencia de la flora endógena de sus $\mathrm{UCl}$.

\section{Quinta norma. «Seleccionar un tratamiento dirigido cuando se conozca la etiología de la infección»}

La información obtenida en los servicios de microbiología es la base del tratamiento dirigido. El aislamiento de uno o más microorganismos en alguna de las muestras de seguridad (sangre, LCR, líquido pleural, exudados purulentos obtenidos por punción, etc.) permite readaptar el tratamiento inicial. Siempre que sea posible, se deben escoger los antibióticos con el espectro de actividad más seguro y reducido, con evidencias contrastadas de su eficacia clínica y microbiológica, de su tolerabilidad así como de una mejor relación coste-beneficio.

En la mayoría de las infecciones en las que se conoce el patógeno causante de la infección, el tratamiento puede realizarse utilizando un solo antibiótico (monoterapia). En aquellos casos de microorganismos en los que es posible la aparición rápida de resistencias durante el tratamiento o en los que se ha documentado una proporción elevada de fracasos terapéuticos con monoterapia, se recomienda el empleo de 2 o más antimicrobianos ${ }^{29}$. Cuando se aíslan 2 o más agentes patógenos en una misma muestra, debe valorarse la importancia de cada uno de los microorganismos identificados (alguno de ellos puede contaminar la muestra o proceder de una colonización). Si se acepta la etiología polimicrobiana en una determinada infección, se debe intentar una cobertura global con el mínimo número de antibióticos.

\section{Sexta norma. «Monitorizar la eficacia del tratamiento»}

La utilización de antibióticos no debe ser un acto sistemático que tranquiliza al médico, sino que debe acompañarse de un conjunto de medidas activas para vigilar la eficacia de estos. Se recomienda hacer la primera valoración de la respuesta terapéutica a las $72 \mathrm{~h}$ de iniciado el tratamiento empírico. La aparición de nuevos signos de infección o el empeoramiento de los signos iniciales debe hacer sospechar que los antibióticos que se administran no son adecuados para tratar los agentes patógenos causantes de la infección. En este caso se repetirá la obtención de muestras de sangre y de los tejidos infectados y se procederá a la administración de antibióticos de rescate utilizando otros más potentes, de mayor espectro y con cobertura para patógenos potencialmente multirresistentes. En el caso contrario, en el que se observa una disminución de los signos iniciales, se continuará el tratamiento hasta la identificación de los patógenos y su sensibilidad, en cuyo caso se procede a su ajuste, tal como se ha indicado anteriormente.

En los casos en los que el tratamiento sea adecuado (según antibiograma) y la evolución no sea favorable, es necesario comprobar que los antibióticos que se administran tienen una buena penetración en los tejidos infectados, que se dan a las dosis adecuadas o que se dan con los intervalos necesarios para asegurar una relación farmacocinética/ farmacodinámica $(\mathrm{pK} / \mathrm{pD})$ adecuada ${ }^{30,31}$.

Para hacer la valoración del tratamiento se debe atender a la respuesta clínica y microbiológica, tanto al finalizar el tratamiento como en la visita de seguimiento, que puede oscilar entre 7-60 días dependiendo de la infección tratada.

\section{Séptima norma. «Vigilar la aparición de efectos adversos o flora emergente multirresistente»}

Cada familia de antibióticos se ha asociado con efectos adversos específicos. Muchos de estos son comunes a más de una familia de antibióticos y algunos se potencian con la 
utilización de otros productos farmacológicos, por lo que en la mayoría de las ocasiones es difícil atribuir a un fármaco un determinado efecto adverso.

Algunos de los efectos adversos más frecuentes (toxicidad renal, toxicidad ótica, selección de cepas mutantes resistentes) se relacionan con concentraciones plasmáticas inadecuadas de los antibióticos ${ }^{32,33}$. Los pacientes críticos, en especial los quirúrgicos complicados, los quemados y los cardiópatas descompensados, presentan con frecuencia un importante aumento del volumen de distribución corporal, lo que influye en las concentraciones plasmáticas o tisulares alcanzadas. La inestabilidad hemodinámica y el fracaso renal condicionan, asimismo, la eliminación de los antibióticos. Estas características modifican el comportamiento farmacocinético de los antibióticos y justifican la amplia variabilidad interindividual en los niveles séricos obtenidos cuando se administran las mismas dosis. Por eso, es conveniente determinar las concentraciones plasmáticas de los antibióticos, en especial la de aquellos con un margen terapéutico pequeño (diferencia entre concentraciones tóxicas y concentraciones terapéuticas), como los aminoglucósidos y la vancomicina. La incorporación de programas de farmacocinética diseñados específicamente para la monitorización de estos fármacos permite ajustar su dosificación para obtener la máxima eficacia clínica con la mínima incidencia de efectos adversos ${ }^{34}$.

El consumo de antibióticos en las UCI facilita la aparición de microorganismos patógenos multirresistentes, cuya presencia puede asociarse con fracaso del tratamiento administrado en un paciente concreto y con cambios en la política de antibióticos de esa $\mathrm{UCl}^{35-38}$. Esto justifica la realización de vigilancia epidemiológica en los pacientes con utilización prolongada de antibióticos, que incluye la obtención de muestras en el foco de infección y en las mucosas (orofaringe, tráquea, heces).

\section{Octava norma. «Limitar la duración del tratamiento} en función de la respuesta clínica o microbiológica»

No existen indicaciones precisas sobre la duración del tratamiento de las infecciones en pacientes críticos. La respuesta clínica y microbiológica al tratamiento, la etiología de la infección y las características de los pacientes (inmunodepresión, prótesis, dispositivos intravasculares) son los principales factores para tener en cuenta al decidir la duración del tratamiento. La mayoría de las infecciones presentes en los pacientes críticos precisan de tratamiento antibiótico durante el tiempo necesario para que desaparezcan los signos y los síntomas clínicos más importantes de la infección, como son fiebre, leucocitosis, inestabilidad hemodinámica, intolerancia al aporte de glucosa y shunt pulmonar. A las $48-72 \mathrm{~h}$ de controlarse estos síntomas puede retirarse el tratamiento antimicrobiano. La duración del tratamiento en pacientes no inmunodeprimidos con sepsis por bacilos gramnegativos oscila entre 8-14 días. Cuando las infecciones están producidas por patógenos multirresistentes, en los que existen evidencias de recidivas, como P. aeruginosa, Acinetobacter spp., S. aureus resistentes a meticilina o enterobacterias productoras de betalactamasas de espectro extendido, el tratamiento debe prolongarse por lo menos hasta las 2 semanas $^{39}$.
La persistencia de patógenos en la vía aérea de pacientes con traqueostomía o ventilación mecánica prolongada, en ausencia de signos clínicos de infección, no debe ser motivo de prolongación del tratamiento. Las infecciones urinarias relacionadas con sonda uretral producidas por bacilos gramnegativos y cocos grampositivos suelen responder, en la mayoría de los casos, a una semana de tratamiento. Cuando la evolución clínica y los estudios microbiológicos descartan la presencia de una infección o existe la evidencia de un diagnóstico alternativo, deben retirarse los antibióticos.

\section{Novena norma. «Responsabilizar a un médico intensivista del control, la vigilancia y el tratamiento de las infecciones»}

En las $\mathrm{UCl}$, la figura del médico intensivista con dedicación parcial al control y el tratamiento de infecciones así como al control de la utilización de antibióticos ha supuesto un impulso en la consolidación de la política de antibióticos en estas áreas de alto riesgo. El paso previo para esto ha sido reconocer que uno de los objetivos de cualquier servicio, incluida la $\mathrm{UCl}$, es monitorizar la morbilidad que se genera con su actividad. Entre los indicadores de calidad de un servicio, reconocidos por los diferentes responsables de la sanidad pública, se incluye el conocimiento de la evolución de las infecciones relacionadas con la asistencia sanitaria así como del uso y el consumo de antibióticos.

Las funciones del médico intensivista especializado en el tratamiento de pacientes críticos con infecciones graves son las siguientes:

1) Conocer y dar a conocer la información obtenida con los sistemas de vigilancia de infección relacionada con la asistencia sanitaria. En colaboración con otros profesionales del hospital, debe participar en los estudios (prevalencia o incidencia) de vigilancia de infección relacionada con la asistencia sanitaria del hospital, pero es el responsable del seguimiento de los enfermos ingresados en $\mathrm{UCl}$. La información de este servicio debe transmitirse de forma regular al comité de infecciones y a la dirección médica o asistencial del hospital pero, al mismo tiempo, debe comunicarse al resto del personal sanitario de la UCl.

2) Mantener relaciones fluidas con los servicios de microbiología y de farmacia. Si la estructura del hospital lo permite, debe participar en las reuniones del servicio de microbiología, en donde se comentan los aislamientos más significativos del hospital. En colaboración con este servicio, debe elaborar el mapa epidemiológico de la UCI en donde se informa no solo de las tasas de las principales infecciones, sino de la evolución de los principales marcadores de multirresistencia y de los patrones de sensibilidad de los agentes patógenos más frecuentes en cada medio. En colaboración con el servicio de farmacología, debe controlar la utilización de los antimicrobianos, tanto en lo que se refiere a indicaciones como a dosis y a duración del tratamiento. Es el interlocutor con estos servicios en las 2 direcciones: informa de la situación de pacientes de riesgo y recibe los datos de mayor interés con la mayor rapidez. 
3) Proponer al personal de la UCI la implantación de protocolos terapéuticos y preventivos para las infecciones más frecuentes. Para esto, debe presentar en el propio servicio los protocolos terapéuticos o de prevención que se elaboran en el hospital, así como aquellos protocolos específicos de la $\mathrm{UCl}$ (prevención de la neumonía en pacientes ventilados y de las infecciones relacionadas con catéteres, entre otros). Siempre que sea posible, debe organizarse un grupo de trabajo en el que colaboren otros profesionales sanitarios de la $\mathrm{UCl}$ (enfermería, auxiliares) con la intención de colaborar conjuntamente en la recogida de información, en el control de la aplicación de los diferentes protocolos y en la revisión periódica de las técnicas y procedimientos realizados por enfermería.

\section{Décima norma. «Corresponsabilizar a todo el personal sanitario del adecuado cumplimiento de las normas»)}

El cumplimiento de las normas de política de antibióticos es responsabilidad de todo el personal médico que trabaja o atiende pacientes en $\mathrm{UCl}$. La realización de reuniones periódicas en las que se presenten los indicadores de consumo de antibióticos así como la evolución de las tasas de infección y de los patrones de sensibilidad de los patógenos más frecuentes permite la revisión de los protocolos de actuación y corresponsabilizar a todos los médicos para su cumplimiento. La realización de controles y auditorías en las que se investiga el cumplimiento de un determinado protocolo asistencial permite conocer el grado de seguimiento de las normas de política de antibióticos en un determinado servicio y por unos determinados profesionales.

\section{Estrategias generales del uso de antibióticos en pacientes críticos}

Con la finalidad de optimizar el uso de antibióticos en el entorno de los pacientes críticos se han propuesto diversas estrategias. Entre ellas destacan la desescalada terapéutica (de-escalation therapy), el ciclado de antibiótico (antibiotic cycling), el tratamiento anticipado (preemptive therapy) y la aplicación de parámetros $\mathrm{pK} / \mathrm{pD}$ para ajustar la dosificación.

1) Desescalada terapéutica (de-escalation therapy). Consiste en la administración inicial de un amplio tratamiento empírico con la intención de cubrir los patógenos más frecuentemente relacionados con la infección por tratar, incluidos los patógenos multirresistentes, seguido de un ajuste rápido del tratamiento antibiótico una vez conocido el agente etiológico ${ }^{40}$. El ajuste debe realizarse entre el segundo y tercer día de la instauración del tratamiento antibiótico inicial. La aplicabilidad de esta estrategia se ha evaluado principalmente en pacientes críticos con neumonía nosocomial o shock séptico ${ }^{41-45}$. El objetivo de la desescalada terapéutica es lograr una menor morbimortalidad al conseguir de forma precoz un tratamiento empírico adecuado, al mismo tiempo que limitar la aparición de resistencias bacterianas por una menor presión antibiótica. Lamentablemente, ninguno de los estudios realizados hasta el presente ha podido demostrar los objetivos buscados ${ }^{41-45}$. Asimismo, la aplicabilidad de esta estrategia obliga a cumplir unas condiciones mínimas que no están disponibles en todas las $\mathrm{UCl}$, como son: a) el conocimiento del mapa epidemiológico del ecosistema bacteriano, incluido el patrón de sensibilidad de los patógenos mas frecuentes; b) una rápida respuesta de los estudios microbiológicos, y c) el cumplimiento de la recomendación de ajustar el tratamiento empírico inicial a los datos microbiológicos de seguridad.

2) Ciclado de antibióticos (antibiotic cycling). Consiste en la sustitución periódica de una clase de antibiótico por otra clase o la combinación de antibióticos que presentan un espectro de actividad similar, pero que no comparten el mismo mecanismo de resistencia. Durante cada período o ciclo, de duración de entre unas semanas y pocos meses, solo pueden utilizarse aquellos antibióticos correspondientes a ese ciclo. De esta forma se pretende disminuir la aparición de resistencias al sustituir el antibiótico antes de que estas se produzcan y preservar su actividad para volver a introducirlo en el hospital en un ciclo posterior.

Esta estrategia, que tiene su origen en la década de $1980^{46}$, se ha propuesto en los últimos años para prevenir el desarrollo de patógenos multirresistentes en el entorno de pacientes críticos. Los estudios realizados hasta el momento han tenido resultados contradictorios, aunque las numerosas diferencias entre ellos no permiten establecer comparaciones ${ }^{47-51}$. Las limitaciones metodológicas de muchos de ellos y la imposibilidad de controlar otras variables que pueden influir en los resultados (ingreso de pacientes con patógenos multirresistentes, adherencia a las pautas de ciclado) impiden evaluar esta estrategia ${ }^{52-55}$.

3) Tratamiento anticipado (preemtive therapy). Consiste en la administración de antimicrobianos en determinados pacientes antes de que aparezcan los signos clínicos de infección. El concepto fue desarrollado en pacientes hematológicos a partir de la aplicación de pruebas serológicas, lo que permitía avanzar el diagnóstico de la infección antes de la aparición de signos clínicos ${ }^{56}$. Su aplicación a pacientes críticos se basa en la identificación de pacientes de riesgo de infecciones asociadas con alta mortalidad, como son las candidemias o candidiasis invasoras. El desarrollo de diferentes scores clínicos ${ }^{57,58}$ ha permitido identificar a estos pacientes, aunque hasta el momento no existe ningún estudio que demuestre una disminución de la mortalidad con la aplicación de esta estrategia en la UCI.

4) Parámetros $p K / p D$. Tradicionalmente se ha recomendado la determinación de niveles plasmáticos de aquellos antibióticos con margen terapéutico estrecho, en los que las concentraciones terapéuticas se aproximan a las tóxicas. Esta situación se produce, principalmente, en el caso de los aminoglucósidos y la vancomicina ${ }^{55}$. Sin embargo, en los últimos años, se ha demostrado la importancia clínica (respuesta clínica, desarrollo de cepas mutantes) de diferentes relaciones farmacodinámicas que se establecen entre las concentraciones plasmáticas de los antibióticos y la concentración mínima inhibitoria (CMI) de las bacterias. Entre ellas destacan la 
relación entre la concentración máxima plasmática y la CMI, el área bajo la curva y la CMI y el porcentaje de tiempo (intervalo de dosis) en el que la concentración plasmática está por encima de la $\mathrm{CMI}^{59}$. La obtención de unos determinados valores de los indicadores anteriores se ha asociado con mayor efectividad del antibiótico (aumentar el poder bactericida y la lisis de las bacterias así como disminuir la aparición de cepas multirresistentes) ${ }^{60-63}$. En los pacientes críticos ingresados en $\mathrm{UCI}$, la determinación de niveles plasmáticos de antibióticos está especialmente indicada por la presencia de diferentes factores que modifican el comportamiento farmacocinético de estos fármacos y justifican la amplia variabilidad interindividual en los niveles séricos obtenidos cuando se administran dosis similares. Lamentablemente, no existen estudios clínicos que demuestren la eficacia de esta estrategia para disminuir la mortalidad de los pacientes críticos y existen múltiples problemas técnicos en la mayoría de los hospitales para su aplicación en la práctica diaria ${ }^{64}$.

\section{Conclusiones}

La política de antibióticos aplicada a pacientes críticos debe basarse en el cumplimiento de un conjunto de normas básicas de uso de los antibióticos. Las estrategias propuestas en los últimos años para optimizar su efectividad y minimizar los efectos adversos deben aplicarse con cautela, se deben evaluar en todo momento los resultados conseguidos y adaptarlos a las necesidades de cada $\mathrm{UCl}$ en particular. Los programas de educación a los clínicos en el cumplimiento de las normas básicas de uso de antibióticos y la desescalada terapéutica sobre la base de los resultados del cultivo microbiológico son las mejores opciones para obtener el éxito en el control de las resistencias a antibióticos.

\section{Bibliografía}

1. Álvarez-Lerma F, ICU-Adquired Pneumonia Study Group. Modification of empiric antibiotic treatment in patients with pneumonia acquired in intensive care unit. Intensive Care Med. 1996;22:387-94.

2. Luna CL, Vujacich P, Niederman MS, Vay C, Gherardi C, Matera J, et al. Impact of BAL data on the therapy ant outcome of ventilator-associated pneumonia. Chest. 1997;111:676-85.

3. Kollef $\mathrm{MH}$. Inadequate antimicrobial treatment of infections: A risk factor for hospital mortality among critically ill patients. Chest. 1999;115:462-74.

4. Ibrahim EH, Sherman G, Ward S, Fraser VJ, Kollef MH. The influence of inadequate antimicrobial treatment of bloodstream infections on patient outcomes in the ICU setting. Chest. 2000;118:146-55.

5. Rahal JJ, Urban C, Horn D, Freeman K, Segal-Maurer J, Mariano $\mathrm{N}$, et al. Class restriction of cephalosporin use to control total cephalosporin resistance in nosocomial Klebsiella. JAMA. 1998;14:1233-7.

6. Fridkin SK, Edwards JR, Tenover FC, Gaynes RP, McGowan Jr JE, Intensive Care Antimicrobial Resistance Epidemiology (ICARE) Project; National Nosocomial Infections Surveillance (NNIS) System Hospitals. Antimicrobial resistance prevalence rates in hospital antibiograms reflect prevalence rates among pathogens associated with hospital-acquired infections. Clin Infect Dis. 2001;33:324-30.

7. Álvarez-Lerma F, Palomar M, Grau S. Management of antimicrobial use in the intensive care unit. Drugs. 2001;61:763-75.

8. Álvarez-Lerma F, Olaechea P, Grau S, Marín M, Domínguez A, Martínez-Lanao J, et al. Grupo de Estudio de Infección en el paciente crítico (GEIPC-SEIMC). Recomendaciones para la monitorización de antibióticos en pacientes de la UCI. Enferm Infecc Microbiol Clin. 2008;26:230-9.

9. Díaz E, Rello J. Top ten list in antibiotic policy in the ICU. Chest. 2002;122:712-4.

10. American College of Chest Physicians and Society of Critical Care Medicine Consensus Conference: Definitions for sepsis and organ failure and guidelines for use of innovative therapies in sepsis. Crit Care Med. 1992;20:864-74.

11. European Society of Intensive Care Medicine. The problem of sepsis- an expert report of the European Society of Intensive Care Medicine. Intensive Care Med. 1994;20:300-4.

12. Vandenbroucke-Grauls CMJE, Vandenbroucke JP. Effect of selective decontamination of the digestive tract on respiratory tract infections and mortality in the intensive care unit. Lancet. 1991;338:859-62.

13. Meta-analysis of randomised controlled trials of selective decontamination of the digestive tract. Selective Decontamination of the Digestive Tract Trialists' Collaborative Group. BMJ. 1993;307:525-32.

14. Kollef $\mathrm{MH}$. The role of selective digestive tract decontamination on mortality and respiratory tract infections. A meta-analysis. Chest. 1994;105:1101-8.

15. Silvestri L, Van Saene HK, Milanese M, Gregori D, Gullo A. Selective decontamination of the digestive tract reduces bacterial bloodstream infection and mortality in critically ill patients. Systematic review of randomized, controlled trials. J Hosp Infect. 2007;65:187-203.

16. Silvestri L, Van Saene HK, Thomann C, Perić M. Selective decontamination of the digestive tract reduces pneumonia and mortality without resistance emerging. Am J Infect Control. 2007;35:354-7.

17. Sirvent JM, Torres A, El-Ebiary M, Castro P, De Batlle J, Bonet A. Prospective effect of intravenously administered cefuroxime againts nosocomial pneumonia in patients with structural coma. Am J Respir Crit Care Med. 1997;155:1729-34.

18. Safdar N, Said A, Lucey MR. The role of selective digestive decontamination for reducing infection in patients undergoing liver transplantation: A systematic review and meta-analysis. Liver Transpl. 2004;10:817-27.

19. Tetteroe GW, Wagenvoort JH, Castelein A, Tilanus HW, Ince C, Bruining HA. Selective decontamination to reduce gramnegative colonization and infections after aesophageal resection. Lancet. 1990;335:704-7.

20. Nathens AB, Marshall JC. Selective decontamination of digestive tract in surgical patients. A systematic review of the evidence. Arch Surg. 1999;134:170-6.

21. Pederzoli P, Bassi C, Vesentini S, Campedelli A. A randomized multicenter clinical trial of antibiotic prophylaxis of septic complications in acute necrotizing pancreatitis with imipenem. Surg Gynecol Obstet. 1993;176:480-3.

22. Dellinger EP, Tellado JM, Soto NE, Ashley SW, Barie PS, Dugernier T, et al. Early antibiotic treatment for severe acute necrotizing pancreatitis: A randomized, double-blind, placebocontrolled study. Ann Surg. 2007;245:674-83.

23. Jorda R, Parras F, Ibañez J, Reina J, Bergadá J, Raurich JM. Diagnosis of nosocomial pneumonia in mechanically ventilated patients by the blind protected telescoping catheter. Intensive Care Med. 1993;19:377-83.

24. Pugin J, Auckenthaler R, Mili N, Janssens JP, Lew PD, Suter PM. Diagnosis of ventilator-associated pneumonia by bacteriologic analysis of bronchoscopic and nonbronchoscopic "blind" 
bronchoalveolar lavage fluid. Am Rev Respir Dis. 1991;143: 1121-9.

25. Sánchez Carrillo C, Guerrero Gómez C. Recogida, transporte y procesamiento general de muestras de laboratorio de Microbiología. En: Cercenado E, Cantón R. Procedimientos de Microbiología Clínica. Recomendaciones de la Sociedad Española de Enfermedades Infecciosas y Microbiología Clínica (SEIMC). 2003. [consultado 23/4/2010]. Disponible en: http:// www.seimc.org/documentos/protocolos/microbiologia/.

26. Ribes JA, Limper AH, Espy MJ, Smith TF. PCR detection of Pneumocystis carinii in bronchoalveolar lavage specimens: Analysis of sensitivity and specificity. J Clin Microbiol. 1997;35: 830-5.

27. Sandhu GS, Kline BC, Stockman L, Roberts GD. Molecular probes for diagnosis of fungal infections. J Clin Microbiol. 1995;33: 2913-9.

28. Coll P, Coque MT, Domínguez MA, Vázquez J, Vila J. Métodos moleculares de tipificación epidemiológica en bacteriología. En: Cercenado E, Cantón R. Procedimientos de Microbiología Clínica. Recomendaciones de la Sociedad Española de Enfermedades Infecciosas y Microbiología Clínica (SEIMC). 2005. [consultado 23/4/2010]. Disponible en: http://www.seimc. org/documentos/protocolos/microbiologia/.

29. Fink MP, Snydman DR, Niederman MS, Leeper KV, Johnson RH, Heard SO, et al. Treatment of severe pneumonia in hospitalized patients: Results of a multicenter, randomized, double-blind trial comparing intravenous ciprofloxacin with impipenemcilastatin. Antimicrob Agents Chemother. 1994;38:547-57.

30. Menéndez R, Perpiñá $M$, Torres A. Evaluation of nonresolving and progressive pneumonia. Semin Respir Infect. 2003;18: 103-11.

31. Pea F, Furlanut M. Pharmacokinetic aspects of treating infections in the intensive care unit: Focus on drug interactions. Clin Pharmacokinet. 2001;40:833-68.

32. Foxworth J. Recognizing and preventing antibiotic-associated complications in the critical care setting. Crit Care Nurs Q. 1997;20:1-11.

33. Joannidis M. Drug-induced renal failure in the ICU. Int J Artif Organs. 2004;27:1034-42.

34. Debord J, Voultoury JC, Lachatre G, Favereau JP, Gay R. Pharmacokinetics and dosage regimens of amikacin in intensive care unit patients. Int J Biomed Comput. 1994;36:135-7.

35. Dworzak DL, Pugsley MP, Sanders CL, Horowitt EA. Emergence of resistance in Gram-negative bacteria during therapy with extended-spectrum cephalosporin. Eur J Clin Microbiol. 1987;6:456-9.

36. Neuhauser MM, Weinstein RA, Rydman R, Danziger LH, Karam G, Quinn JP. Antibiotic resistance among Gram-negative bacilli in US Intensive Care Units. Implications for fluoroquinolone use. JAMA. 2003;289:885-8.

37. Akinci E, Colpan A, Bodur H, Balaban N, Hervía A. Risk factors for ICU-acquired imipenem-resistant gram-negative bacterial infections. J Hosp Infect. 2005;59:317-23.

38. Meyer E, Schwab F, Gastmeier P, Rueden H, Daschner FD, Jonas D. Stenotrophomonas maltophilia and antibiotic use in German intensive care units: Data from Project SARI (Surveillance of Antimicrobial Use and Antimicrobial Resistance in German Intensive Care Units. J Hosp Infect. 2006;64:238-43.

39. Chastre J, Wolff M, Fagon JY, Chevret S, Thomas F, Wermert D. Comparison of 8 vs 15 days of antibiotic therapy for ventilatorassociated pneumonia in adults: a randomized trial. JAMA. 2003;290:2588-98.

40. Kollef MH. Hospital-acquired pneumonia and de-escalation of antimicrobial treatment. Crit Care Med. 2001;29:1473-5.

41. Ibrahim EH, Ward S, Sherman G, Schaiff R, Fraser VJ, Kollef MH. Experience with a clinical guideline for the treatment of ventilator-associated pneumonia. Crit Care Med. 2001;29: 1109-15.
42. Rello J, Vidaur L, Sandiumenge A, Rodríguez A, Gualis B, Boque $C$, et al. De-escalation therapy in ventilator-associated pneumonia. Crit Care Med. 2004;32:2183-90.

43. Álvarez-Lerma F, Álvarez B, Luque $\mathrm{P}$, Ruiz F, Domínguez-Roldán $J M$, Quintana E, et al. Empiric broad-spectrum antibiotic therapy of nosocomial pneumonia in the intensive care unit: A prospective observational study. Crit Care. 2006;10:R78.

44. Leone M, Garcin F, Bouvenot J, Boyadjev I, Visintini P, Albanèse J, et al. Ventilator-associated pneumonia: Breaking the vicious circle of antibiotic overuse. Crit Care Med. 2007;35:379-85.

45. Giantsou E, Liratzopoulos N, Efraimidou E, Panopoulou M, Alepopoulou E, Kartali-Ktenidou S, et al. De-escalation therapy rates are significantly higher by bronchoalveolar lavage than by tracheal aspirate. Intensive Care Med. 2007;33:1533-40.

46. Gerding DN, Larson TA, Hughes RA, Weiler M, Shanholtzer C, Peterson LR. Aminoglycoside resistance and aminoglycoside usage: Ten years of experience in one hospital. Antimicrob Agents Chemother. 1991;35:1284-90.

47. Kollef MH, Vlasnik J, Sharpless L, Pasque C, Murphy D, Fraser V. Scheduled change of antibiotic classes. A strategy to decrease the incidence of ventilator-associated pneumonia. Am J Respir Crit Care Med. 1997;156:1040-8.

48. Gruson D, Hibert G, Vargas F, Valentino R, Bebear C, Allery A, et al. Rotation and restricted use of antibiotics in a medical intensive care unit: Impact on the incidence of ventilatorassociated pneumonia caused by antibiotic-resistant gramnegative bacteria. Am J Respir Crit Care Med. 2000;162:837-43.

49. Hughes MG, Evans HL, Chong TW, Smith RL, Raymond DP, Pelletier SJ, et al. Effect of an intensive care unit rotating empiric antibiotic schedule on the development of hospitalacquired infections on the non-intensive care unit ward. Crit Care Med. 2004;32:53-60.

50. Merz LR, Warren DK, Kollef MH, Fraser VJ. Effects of an antibiotic cycling program on antibiotic prescribing practices in an intensive care unit. Anitmicrob Agents Chemother. 2004;48: 2861-5.

51. Martínez JA, Nicolás JM, Marco F, Horcajada JP, García-Segarra $G$, Trilla A, et al. Comparison of antimicrobial cycling and mixing strategies in two medical intensive care units. Crit Care Med. 2006;34:329-36.

52. Sandiumenge A, Díaz E, Rodríguez A, Vidaur L, Canadell L, Olona $\mathrm{M}$, et al. Impact of diversity of antibiotic use on the development of antimicrobial resistance. J Antimicrob Chemother. 2006;57:1197-204.

53. Brown E, Nathwani D. Antibiotic cycling or rotation: A systematic review of the evidence of efficacy. J Antimicrob Chemother. 2005;55:6-9.

54. Masterton RG. Antibiotic cycling: more than it might seem? J Antimicrob Chemother. 2005;55:1-5.

55. Kollef MH. Is antibiotic cycling the answer to preventing the emergence of bacterial resistance in the Intensive Care Unit? Clin Infect Dis. 2006;43:S82-8.

56. Maertens J, Theunissen K, Verhoef G, Verschakelen J, Lagrou K, Verbeken $\mathrm{E}$, et al. Galactomannan and computed tomographybased preemptive antifungal therapy in neutropenic patients at high risk for invasive fungal infection: A prospective feasibility study. Clin Infect Dis. 2005;41:1242-50.

57. Pittet D, Monod M, Suter PM, Frenk E, Auckenthaler R. Candida colonization and subsequent infections in critically ill surgical patients. Ann Surg. 1994;220:751-8.

58. León C, Ruiz-Santana S, Saavedra P, Almirante B, Nolla-Salas J, Álvarez-Lerma $\mathrm{F}$, et al. A bedside scoring system ("Candida score") for early antifungal treatment in nonneutropenic critically ill patients with Candida colonization. Crit Care Med. 2006;34:730-7.

59. Blondeu JM, Hansen G, Metzler K, Hedlin P. The role of PK/PD parameters to avoid and increase of resistance: Mutant prevention concentration. J Chemother. 2004;16:1-19. 
60. Preston SL, Drusano GL, Berman AL, Fowler CL, Chow AT, Dornseif B, et al. Pharmacodynamics of levofloxacin: A new paradigm for early clinical trials. JAMA. 1998;279:159-60.

61. Forrest A, Nix DE, Ballow CH, Goss TF, Birmingham $M$, Schentag JJ. Pharmacodynamics of intravenous ciprofloxacin in seriously ill patients. Antimicrob Agents Chemother. 1993;37: 1073-81.

62. Thomas JK, Forrest A, Bhavnani SM, Hyatt JM, Cheng A, Ballow $\mathrm{CH}$. Pharmacodynamic evaluation of factors associated with development of bacteria resistance in acutely ill patients during therapy. Antimicrob Agents Chemother. 1998;42:521-7.

63. Pea F, Furlanut M. Pharmacokinetic aspects of treating infections in the intensive care unit: Focus on drug interactions. Clin Pharmacokinet. 2001;40:833-68.

64. Grau S, Álvarez-Lerma F, Domínguez-Gil A. Pharmacokinetic/ pharmacodynamic indices: Are we ready to use them in daily practice? Expert Rev Anti Infect Ther. 2007;5:913-6. 